fehlen, zu jedem Land einige Daten und Fakten in standardisierter Form zu präsentieren, zum Beispiel die Namen der gewählten Präsidenten und die Wahlergebnisse seit der Anfang der 1980er Jahre einsetzenden Re-Demokratisierung.

Auf ein abschließendes Kapitel haben die Herausgeber - sinnvollerweise - verzichtet. Dies würde der Vielfalt auf dem lateinamerikanischen Kontinent, der in den Jahren 2004 bis 2008 durch wirtschaftliche Dynamik gekennzeichnet war und eine sehr viel jüngere Bevölkerung aufweist als Europa, nicht gerecht. Es ist auch kein Nachteil, dass sich die Autoren auf die geschichtlichen und politischen Aspekte konzentrieren und auf knapp 600 Seiten gar nicht erst den Versuch machen, die sozialen und wirtschaftlichen Probleme von fast 900 Mio. Menschen zu erklären. Sowohl in der Einleitung als auch in den meisten Länderartikeln wird das Bekenntnis zur Demokratie als eine Gemeinsamkeit von Nordund Lateinamerika betrachtet. Ob in späteren Ausgaben diese optimistische Sichtweise Bestand haben wird, kann erst die zukünftige Entwicklung zeigen. Aufgrund der sowohl historisch als auch politikwissenschaftlich ausgerichteten Konzeption des Bandes dürfte es möglich sein, in einer Neuausgabe auch den aktuellen Trend hin zu plebiszitären und populistischen Formen der Demokratie gerade in Südamerika zu berücksichtigen.

Nikolaus Werz

\title{
Informative Einführungen in die sechs politischen Systeme Ostasiens
}

Heberer, Thomas und Claudia Derichs (Hrsg.): Einführung in die politischen Systeme Ostasiens. VR China, Hong Kong, Japan, Nordkorea, Südkorea, Taiwan, VS Verlag für Sozialwissenschaften, 2. aktualisierte und erweiterte Auflage, Wiesbaden 2008, 463 Seiten, € 34,90.

Die Länder Ostasiens sind zum neuen Gravitationszentrum der Weltwirtschaft geworden und werden deshalb oft als ein einheitlicher Block betrachtet. Es heißt, dass sie alle zum so genannten „konfuzianischen Kulturkreis“ gehören und auch die Geschichte teilen, dass sie im 19. Jahrhundert gezwungen waren, sich in eine vom Westen dominierte internationale Weltordnung einzufügen. Dabei wird leicht die Tatsache ignoriert, dass die „konfuzianische Kultur" als gemeinsame kulturelle Wurzel dieser Region umstritten ist. Zudem vergisst man, dass diese Staaten im 20. Jahrhundert nicht nur eine rasante wirtschaftliche Entwicklung, sondern auch den radikalen Wandel ihrer politischen Systeme, ja tief greifende Brüche in ihren politischen Verhältnissen erlebt haben: von der traditionellen Monarchie zur Demokratie beziehungsweise zum Sozialismus. Zugleich wurden sie, durchaus auch mit eigenem Zutun, in die Weltkriege und in den Kalten Krieg hineingezogen, der in Ostasien bis heute nicht beendet ist, wie in der Teilung der koreanischen Halbinsel zu sehen ist.

Nicht zuletzt diese komplexen historischen Hintergründe waren verantwortlich dafür, dass bisher nur selten der Versuch unternommen worden ist, die politischen Systeme der Länder Ostasiens in ihrem gesamten Umfang zu untersuchen. Mit diesem von Thomas Heberer und Claudia Derichs herausgegebenen Band hat man endlich auch im deutschsprachigen Raum ein respektables Buch, das sich für Einführungsseminare in die politischen Systeme Ostasiens eignet. Seine einzelnen Artikel beruhen auf langjährigen wissenschaftlichen Auseinandersetzungen der Autoren mit ihrem Untersuchungsgegenstand. Dass die 
meisten von ihnen in der Lage sind, landessprachliche Quellen berücksichtigen zu können, ist besonders zu loben. Damit unterscheidet sich der Band von den meisten komparativen Studien zu diesem Thema, die sich hauptsächlich auf englische Quellen stützen.

Das Vorhaben, nach einem einheitlichen Schema die sechs politischen Systeme untersuchen zu wollen, wie es Thomas Heberer in seiner Arbeit über China exemplarisch getan hat, wurde leider nicht in allen Beiträgen umgesetzt. Damit wären interessante Vergleiche ermöglicht worden. So beschränkt sich die vergleichende Analyse im Schlusskapitel auf recht vage Hinweise auf Gemeinsamkeiten der untersuchten Ländern.

Ohne Zweifel erhalten Studienanfänger und Nichtkenner dieser Region einen guten Einblick in die politischen Systeme. Abgesehen von ein paar Flüchtigkeitsfehlern (zum Beispiel wird dem südkoreanischen Präsidenten Roh Moo-Hyun der Familienname Moo beigelegt, S. 301) sind die Studien informativ und leserfreundlich geschrieben. Dieser Band sollte nicht zuletzt deshalb zur Pflichtlektüre für alle Studenten werden, die sich mit Ostasien beschäftigen.

Eun-Jeung Lee

\section{Verschenkte Gelegenheit, Parlamentarismustheorie und Ideengeschichte analytisch zusammenzuführen}

Turkka, Tapani: The Origins of Parliamentarism. A Study of Sandys' Motion (Studies in Political Theory, hrsg. v. Rainer Schmalz-Bruns und Hubertus Buchstein, Bd. 2), Nomos Verlagsgesellschaft, Baden-Baden 2007, 236 Seiten, € 39,-.

Der Titel dieses Buchs weckt falsche Erwartungen: Nicht um die Ursprünge des Parlamentarismus geht es (nichts ist zu lesen über die Synhedrien der hellenistischen Bundesrepubliken, nichts über die Konzilien der Kirche, auch nichts über den Ursprung jener ständischen Vertretungskörperschaften, aus denen der moderne Parlamentarismus entstand), sondern behandelt werden allein die Ursprünge des parlamentarischen Regierungssystems in der Ära von Sir Robert Walpole (1721 bis 1742). So formuliert es Tapani Turkka auf S. 216 denn auch im ersten Satz seiner Zusammenfassung. Die Umstände, in denen damals das parlamentarische Regierungssystem als „government by corruption“ entstand; erste Konzeptionen von parlamentarischer Opposition; auch die Vorstellung, eine Regierung abzulösen, sei nicht erst aufgrund von ihr zugeschriebenen rechtlichen Verfehlungen legitim, sondern einfach aus politischen Gründen: Über das alles wird man recht gut informiert - wenn auch im Kern nicht über das hinausgehend, was man etwa schon von Kurt Kluxen und Gerhard A. Ritter kennt.

Ausgangs- sowie Endpunkt der Darstellung ist der letzte und systematisch bedeutendste einer ganzen Reihe von Anträgen des Unterhausabgeordneten Samuel Sandys (1695 bis 1770), Kernelemente von Walpoles Regierungspraxis zu beseitigen. Am 13. Februar 1741 beantragte er, das Unterhaus möge eine Bitte an den König beschließen, den - längst machtmäßig ersten - Minister Walpole aufgrund seiner Regierungsweise und wegen begründeter Unzufriedenheit mit deren Ergebnissen zu entlassen. Dieser Antrag wurde zwar abgelehnt; ein knappes Jahr später trat Walpole aber von sich aus zurück. Mit Sandys'Antrag 